\section{MicroARN et translocations chromosomiques dans les hémopathies malignes}

Marina Bousquet, Nicole Dastugue, Pierre Brousset
Inserm, U563,

Centre de physiopathologie

de Toulouse Purpan, Place Baylac,

31300 Toulouse, France.

brousset.p@chu-toulouse.fr

localisation des microARN au niveau de régions fréquemment altérées dans les cancers. On distingue les microARN suppresseurs de tumeur et les «onco$m i R »$ (microARN oncogènes). En effet, certains microARN contrôlent l'expression d'oncogènes, et leur répression conduit à la surexpression de cet oncogène (ex: le miARN let-7 et l'oncogène RAS), ce sont les TSmiR (tumor suppressor miRNA). En revanche, d'autres (les oncomiR) sont surexprimés dans les cancers et sont susceptibles d'inhiber l'expression de gènes suppresseurs de tumeurs [9]. Les mécanismes dérégulant l'expression de ces microARN sont nombreux et peuvent être corrélés aux mécanismes classiques de dérégulation de gènes: amplifications, translocations chromosomiques, délétions,

Figure 1. MicroARN et translocations chromosomiques dans les hémopathies malignes. A. Implication directe: la $\mathrm{t}(8 ; 17)$ conduit à la surexpression de l'oncogène $c-M Y C$ qui se retrouve sous le contrôle du promoteur de miR142. L'insertion (ins) de la région chromosomique de miR-125b au niveau du locus des IGH (chaîne lourde des immunoglobulines) conduit probablement à la surexpression de miR-125b. La $\mathrm{t}(2 ; 11)$ conduit à la surexpression de miR125b. B. Implication indirecte: les translocations conduisant à la perte de la région 3'UTR de HMGA2 entraînent une surexpression de la protéine qui n'est plus sous le contrôle du microARN let-7. C. Dérégulation du microARN miR-223 par la protéine de fusion AMLl-ETO. $D$. La délétion de miR-203 associée à la translocation $B C R-A B L$ entraîne une surexpression de la protéine de fusion oncogénique. 
mutations ponctuelles, dérégulations épigénétiques. Cependant, peu d'études rapportent l'implication des microARN dans les translocations chromosomiques, mais il existe quelques exemples.

\section{Dérégulation directe de microARN} par les translocations chromosomiques Le premier exemple est la translocation $\mathrm{t}(8 ; 17)$ (q24;q22). Cette translocation juxtapose le microARN miR-142 à l'oncogène $M Y C$. Comme dans les translocations impliquant $M Y C$ et le locus des immunoglobulines, MYC se retrouve sous le contrôle du promoteur de miR-142, ce qui entraîne une surexpression de la protéine MYC dans les lymphomes B [1].

Un autre exemple est la juxtaposition du microARN miR-125b au gène de la chaîne lourde des immunoglobulines dans un cas de leucémie aiguë lymphoblastique $B$ [2]. II s'agit d'une insertion ins $(11 ; 14)$ (q23q24,q32). L'ARNm de ce patient n'étant pas disponible, des analyses du niveau d'expression de miR-125b n'ont pas pu être réalisées. Néanmoins, il est probable que ce réarrangement conduit à une surexpression de miR-125b (b-1), comme c'est le cas dans les réarrangements impliquant la chaîne lourde des immunoglobulines.

Le premier exemple de translocation chromosomique conduisant à la surexpression d'un microARN a été rapporté par notre équipe grâce à l'étude de la translocation chromosomique $t(2 ; 11)(p 21 ; q 23)$, retrouvée chez 19 patients atteints de myélodysplasies $(n=9)$ ou de leucémies aiguës myéloblastiques $(n=10)$. Après clonage des points de cassures par FISH (fluorescent in situ hybridization) et LDI-PCR (long distance inverse PCR), nous avons mis en évidence la surexpression d'un microARN situé à proximité relative du point de cassure. Ainsi, le microARN miR-125b-l est surexprimé - d'un facteur 6 à 90 - chez les patients porteurs de la $t(2 ; 11)$. Par des expériences de différenciation in vitro des lignées HL60 et NB4, nous avons pu démontrer que la surexpression de miR-125b dans ces cellules entraînait un blocage de la différenciation myélomonocytaire in vitro. La Figure 2 illustre le blocage de différenciation monocytaire observé en présence de miR-125b par rapport au miR contrôle, dans des cellules HL60 dont la différenciation a été induite par un traitement par le DMSO (diméthylsulfoxyde) [3]. Dans le même travail, nous avons pu montrer que chez certains patients dont les cellules n'exprimaient pas la translocation $t(2 ; 11)$, une amplification du chromosome 21 q entraînait une surexpression massive de miR-125b-2, I'homologue de miR-125b-1 [3].

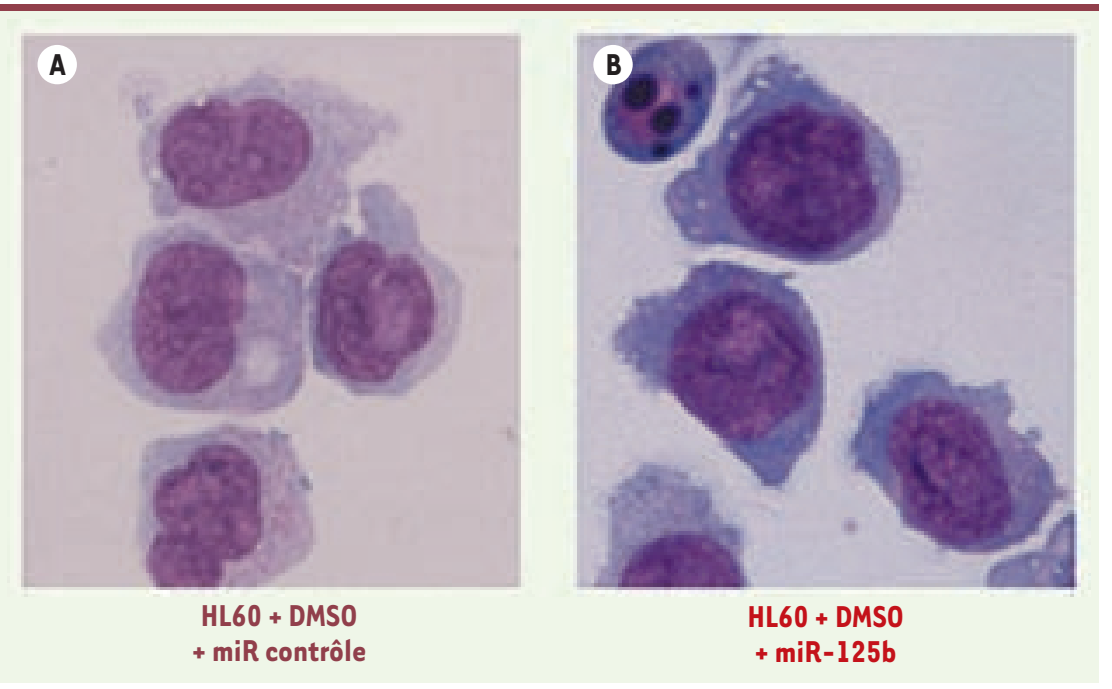

Implication indirecte de microARN dans les translocations chromosomiques L'intervention indirecte d'un microARN dans une translocation a été rapportée dans l'étude des translocations impliquant le gène HMGA2 (high mobility group AT-hook 2). Les réarrangements au niveau du gène HMGA2, situé en $12 q 15$, sont fréquents et retrouvés dans différents cancers (adénomes, carcinome, leucémies, lipomes, sarcomes...). Dans la plupart des cas, les trois domaines de liaisons à l'ADN de HMGA2 sont conservés dans les séquences géniques chimériques et associés à des séquences variées (gènes FHIT, LPP, LHFP, RAD51L1, CMKORl, EBF, RDCl, NFIB, ... $)^{1}$. Cependant, dans certains cas, le point de cassure au sein du gène HMGA2 se situe en dehors de sa région codante et est associé à une forte expression de la protéine [4]. En 2001, Borrmann et al. observent, en utilisant une construction contenant le gène rapporteur luciférase associé à la région 3' non traduite de HMGA2, une sous-expression du gène rapporteur, suggérant la présence d'éléments répresseurs dans la région 3'UTR de HMGA2 [5]. L'étude de Mayr et al. a permis de mieux comprendre ces phénomènes. En effet, ces auteurs ont pu démontrer que HMGA2 est régulé par let 7 qui se lie sur sa partie 3 ' non traduite [6]. Les translocations conduisant à I'élimination de la partie 3' non traduite de HMGA2 empêchent la répression par

${ }^{1}$ FHIT : fragile histidine triad gene; LPP: lipoma preferred partner gene; LFHP: lipoma HMGIC fusion partner gene; CMKORI : chemokine orphan receptor 1 ; $\varepsilon B F$ : early $B$ cell factor gene; NfiB: Nuclear factor I/B ; RAD51L1: Rad51 Like 1 .

Figure 2. Blocage de la différenciation monocytaire des cellules de la lignée HL60 induit par la surexpression de miR-125b. Le traitement par le DMSO de la lignée myéloblastique HL60 induit une différenciation monocytaire. A. Transfection de la lignée HL60 avec le microARN contrôle en présence de DMSO. $\boldsymbol{B}$. Transfection de la lignée HL60 avec miR-125b en présence de DMSO. 
let-7, ce qui conduit à une surexpression de la protéine HMGA2.

\section{Dérégulation des microARN \\ par des protéines de fusion dans les hémopathies malignes}

Un autre exemple d'implication indirecte de microARN dans les translocations chromosomiques a été rapporté dans l'étude de la protéine de fusion AML1-ETO. AMLI code une des sous-unités du Core binding factor (CBF) qui intervient dans I'hématopoï̀se. ETO, pour eight twenty one, code un facteur de transcription putatif, exprimé au niveau du cerveau. La translocation $\mathrm{t}(8 ; 21)$ conduisant à la formation de la protéine de fusion AMLI-ETO est l'anomalie caryotypique la plus fréquente dans les leucémies aiguës myéloblastiques (LAM). Elle est détectée dans $15 \%$ des LAM et représente $40 \%$ du sous-groupe $\mathrm{M}^{2}{ }^{2}$. L'étude de Fazi et al. a permis de démontrer que le microARN miR-223, essentiel à la différenciation myéloïde, était sous-exprimé dans ces pathologies [7]. Par des expériences d'immunoprécipitation de chromatine, les auteurs ont pu démontrer que la protéine de fusion AML1-عTO était capable de se lier au promoteur de miR-223 suggérant une régulation de la transcription de miR-223 par l'oncoprotéine

${ }^{2}$ Le groupe coopératif Franco-Américano-Britannique (FAB) a défini en 1976 puis dans les années qui ont suivi plusieurs classes de LAM (MI à M7) sur des critères morphologiques distingant le degré de maturité des blastes et la prédominance de la lignée, granuleuse, monocytaire, érythroïde ou mégacaryocytaire.
AML1-ETO. Des expériences complémentaires utilisant des constructions contenant le gène rapporteur luciférase sous le contrôle des régions promotrices de miR-223 et des quantités croissantes de AML1-ETO transfectées dans la lignée 293T, ont permis de définir précisément les sites nécessaires à la régulation de la transcription de miR-223 par AMLlहTO. AMLl-ETO recrute des protéines de remodelage de la chromatine, histones déacétylases ou ADN méthyltransférases, entraînant une répression transcriptionnelle de miR-223 et ainsi un blocage de la différenciation [7].

\section{Régulation des protéines}

\section{de fusion par les microARN}

Bueno et coll. ont mis en évidence une hyperméthylation du promoteur de miR203 dans les leucémies exprimant la protéine de fusion $B C R-A B L^{3}$. Or, miR-203 cible l'oncogène $A B L$ et la réexpression de miR-203 dans ces tumeurs entraîne une diminution du taux de la protéine BCR$A B L$ et en conséquence une inhibition de la prolifération de ces cellules [8].

L'implication des microARN dans les cancers est maintenant clairement établie. Cependant, des études supplémentaires sont nécessaires afin de déterminer l'impact de ces dérégulations dans les

${ }^{3} \mathrm{BCR}-\mathrm{ABL}$ est une protéine chimérique née de la translocation $\mathrm{t}(9 ; 22)$ fusionnant le gène $\mathrm{Abl}$ (Abelson, situé normalement sur le chromosome 9) au niveau de la région bcr ou breakpoint cluster region du chromosome 22. Ce gène de fusion est caractéristique de la leucémie myéloïde chronique mais est également rencontré dans certaines leucémies aiguës. hémopathies malignes. L'identification des cibles de ces microARN est également un élément essentiel afin de mieux comprendre les mécanismes oncogéniques et d'identifier de nouvelles cibles thérapeutiques. $\diamond$

microRNA

and chromosomal translocations

in hematologic malignancies

\section{RÉFÉRENCES}

1. Lagos-Puintana M, Rauhut R, Yalcin A, et al. Identification of tissue-specific microRNAs from mouse. Curr Biol 2002 ; 12 : 735-9.

2. Sonoki T, Iwanaga $\varepsilon$, Mitsuya $H$, Asou N. Insertion of microRNA-125b-1, a human homologue of lin-4, into a rearranged immunoglobulin heavy chain gene locus in a patient with precursor B-cell acute lymphoblastic leukemia. Leukemia 2005 ; 19 : 2009-10.

3. Bousquet M, Quelen C, Rosati R, et al. Myeloid cell differentiation arrest by miR-125b-1 in myelodysplasic syndrome and acute myeloid leukemia with the $\mathrm{t}(2 ; 11)(\mathrm{p} 21 ; \mathrm{q} 23)$ translocation. J Exp Med 2008; $11: 2499-506$.

4. Geurts JM, Schoenmakers EF, Van de Ven WJ. Molecular characterization of a complex chromosomal rearrangement in a pleomorphic salivary gland adenoma involving the 3'-UTR of HMGIC. Cancer Genet Cytogenet 1997; 95 : 198-205.

5. Borrmann L, Wilkening S, Bullerdiek J. The expression of HMGA genes is regulated by their 3'UTR. Oncogene $2001 ; 20: 4537-41$.

6. Mayr C, Hemann MT, Bartel DP. Disrupting the pairing between let-7 and Hmga2 enhances oncogenic transformation. Science 2007 ; 315 : 1576-9.

7. Fazi F, Racanicchi S, Zardo P, et al. Epigenetic silencing of the myelopoiesis regulator microRNA-223 by the AML1/ETO oncoprotein. Cancer Cell 2007 ; 12 : 457-66.

8. Bueno MJ, Pérez de Castro I, Gómez de Cedrón, et al. Genetic and epigenetic silencing of microRNA-203 enhances $A B L 1$ and BCR-ABLI oncogene expression. Cancer Cell 2008 ; 6 : 496-506

9. Finoux AL, Chartrand P. Micro-ARN : oncogènes et suppresseurs de tumeurs. Med Sci (Paris) 2008 ; 24 : 1049-54.

10. Puisieux A, Ansieau S. miARN : des petites molécules associées à un grand risque. Med Sci (Paris) 2008 ; $24: 135-6$.
$>$ Grâce à $m / s$, vous vivez en direct

Tarifs d'abonnement M/S - 2009

Abonnez-vous

à Médecine/Sciences les progrès des sciences biologiques et médicales

$$
\begin{gathered}
\text { Bulletin d'abonnement } \\
\text { page } 212 \text { dans ce numéro de } \mathrm{m} / \mathrm{s}
\end{gathered}
$$

